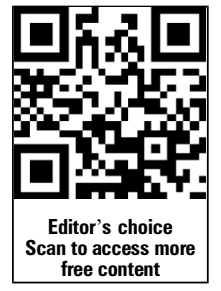

free content
Ophthalmology, University of Cologne, Cologne, Germany

\section{Correspondence to} Dr Thomas S Dietlein, Ophthalmology, University Cologne, Joseph-Stelzmann-Str. 9, Köln D-50931, Germany; Thomas.Dietlein@uk-koeln.de

Received 4 March 2013 Revised 19 May 2013 Accepted 23 May 2013 Published Online First 12 June 2013
To cite: Dietlein TS, Lappas A, Rosentreter A. Br J Ophthalmol 2013;97: 985-988.

\title{
Secondary subconjunctival implantation of a biodegradable collagen-glycosaminoglycan matrix to treat ocular hypotony following trabeculectomy with mitomycin C
}

\author{
T S Dietlein, A Lappas, A Rosentreter
}

\begin{abstract}
Aim Biodegradable collagen glycosaminoglycan matrices (CGM) have been introduced to glaucoma filtration surgery in order to prevent scarring of the filtering bleb. In this retrospective case series, we describe a new surgical concept for treating symptomatic ocular hypotony following filtration surgery with mitomycin $\mathrm{C}$ by a secondary subconjunctival implantation of a CGM.

Methods Depending on bleb morphology, a CGM implant with a diameter of 6 or $12 \mathrm{~mm}$ was placed subconjunctivally on the scleral flap in 12 eyes displaying symptomatic ocular hypotony with $(n=2)$ or without $(n=10)$ bleb leakage. Median period between trabeculectomy and this intervention was 4.5 months (range, 1-72 months).
\end{abstract}

Results Mean intraocular pressure significantly increased from $4.4 \pm 1.4 \mathrm{~mm} \mathrm{Hg}$ to $9.9 \pm 2.8 \mathrm{~mm} \mathrm{Hg}$ $(p<0.0001)$ over a median follow-up of 6 months (range, 2-18 months). Mean visual acuity improved significantly $(p=0.0012)$. Postoperative complications included implant re-exposure in one eye and persistent choroidal detachment requiring resurgery in another eye. Conclusions Subconjunctival implantation of CGM may present an additional surgical tool in the treatment of symptomatic ocular hypotony after filtering surgery. The mechanism of action may be counter-pressure to the scleral flap and secondary wound healing around the CGM scaffold. Long-term observations are needed to fully evaluate this new surgical concept.

\section{INTRODUCTION}

Ocular hypotony following filtering surgery can cause severe visual complications and there are various conservative and surgical treatment options for it. The surgical interventions include injection of viscoelastics into the anterior chamber or bleb, injection of blood into the filtering bleb, transconjunctival flap suturing, open flap resuturing or scleral fistula closure by pericard patches. ${ }^{1-7}$ While injections of viscoelastic and blood usually have little long-lasting effect, techniques to reduce the transcleral flow by suturing or tissue patching can lead to massive elevation of the intraocular pressure (IOP), thus necessitating further pressure-reducing interventions.

There are two potential mechanisms by which a subconjunctival biodegradable implant could help to counteract overfiltration following trabeculectomy with mitomycin C. In the short term, counterpressure exerted by the biodegradable scaffold on the scleral flap may help to limit transscleral flow.
In the midterm and long term, the induction of regular fibroblast ingrowth and improvement of vascularisation, especially in the avascular bleb, ${ }^{8}$ should re-establish sufficient outflow resistance to prevent ocular hypotony. Either mechanism of action is possible with the biodegradable collagenglycosaminoglycan matrix (CGM) that was introduced to ophthalmosurgery several years ago. ${ }^{9-14}$

We here present a retrospective case series of 12 hypotonous eyes that underwent secondary subconjunctival implantation of a porous CGM in the filtering bleb after previous trabeculectomy with mitomycin C.

\section{METHODS}

\section{Patients}

This consecutive case series included 12 glaucoma patients (median age 73 years, range 50-89 years) who had undergone filtration surgery with mitomycin $\mathrm{C}$ and were suffering from subsequent ocular hypotony, with or without bleb leakage. All were treated by using a biodegradable CGM scaffold (Ologen, Aeon Astron Europe BV, The Netherlands). The implant size was either $6 \mathrm{~mm} \times 2 \mathrm{~mm}$ (no. $830601)$ or $12 \mathrm{~mm} \times 1 \mathrm{~mm}$ (no. 830601).

All patients gave informed consent to this procedure after extensive discussion of the different surgical options, according to the tenets of the Declaration of Helsinki.

The median period between trabeculectomy and secondary implantation of CGM was 4.5 months and ranged between 1 and 72 months. All eyes with ocular hypotony (IOP $<8 \mathrm{~mm} \mathrm{Hg}$ ) had either choroidal detachment and/or choroidal folds. In all eyes, visual acuity had dropped compared to the situation before trabeculectomy. Patient characteristics are listed in table 1.

Patients who had religious or other objections against the CGM owing to the porcine origin were excluded from this surgical concept as well as patients with ocular hypotony within the first 3 weeks after trabeculectomy who usually were treated by 'wait-and-see', intraocular injection of viscoelastics or transconjunctival suturing.

\section{Surgical procedure}

The procedure was performed under subconjunctival anaesthesia $(0.5-1.0 \mathrm{~mL}$ Lidocaine $2 \%)$. In the hypotonous eyes without bleb leakage a radial conjunctival incision ( $2 \mathrm{~mm}$ in length) was performed lateral to the filtering bleb, and the subconjunctival space within the filtering bleb area was cautiously 
Table 1 Patient characteristic with preoperative and postoperative data

\begin{tabular}{|c|c|c|c|c|c|c|c|}
\hline \multirow[b]{2}{*}{ Age } & \multirow[b]{2}{*}{ Sex } & \multirow[b]{2}{*}{ Surgical intervention } & \multirow[b]{2}{*}{$\Delta$} & \multicolumn{2}{|l|}{ IOP/VA } & \multirow[b]{2}{*}{$\mathbf{F}$} & \multirow[b]{2}{*}{ Complications } \\
\hline & & & & Preop & Postop & & \\
\hline 69 & $\mathrm{~m}$ & $\begin{array}{l}\text { Phako, Trab/MMC, io visco, } \\
\text { transconjunctival sutures }\end{array}$ & 3 & $5 / 0.4$ & $10 / 1.0$ & 18 & Antiglaucomatous topical medication \\
\hline 89 & $f$ & $\begin{array}{l}\text { Phako, Trab/MMC, io visco, } \\
\text { transconjunctival sutures }\end{array}$ & 6 & $7 / 0.1$ & $14 / 0.6$ & 6 & - \\
\hline 82 & $\mathrm{~m}$ & Trab/MMC, $2 \times$ io visco & 4 & $5 / 0.25$ & $8 / 0.5$ & 6 & - \\
\hline 76 & $f$ & Phako, Trab/MMC, $2 \times$ io visco & 5 & $5 / 0.2$ & $8 / 0.6$ & 12 & - \\
\hline 73 & $\mathrm{~m}$ & Phako, Trab/MMC, laser suture lysis & 2 & $3 / 0.25$ & $13 / 0.25$ & 4 & - \\
\hline 73 & $f$ & Trab/MMC & 1 & $2 / 0.4$ & $9 / 0.6$ & 7 & - \\
\hline 69 & $f$ & Phako, Trab/MMC, io visco & 4 & $5 / 0.5$ & $7 / 1.0$ & 4 & - \\
\hline 80 & $f$ & Phako, trab/MMC, io visco & 1 & $4 / 0.05$ & $11 / 0.4$ & 2 & Additional compression sutures, persisting choroidal detachment, pericard patch \\
\hline 56 & $\mathrm{~m}$ & Trab/MMC & 72 & $6 / 0.1$ & $10 / 0.16$ & 4 & Persisting choroidal folds \\
\hline 76 & f & Trab/MMC & 5 & $3 / 0.4$ & $6 / 0.4$ & 2 & - \\
\hline 63 & $\mathrm{~m}$ & Vitrectomy, Trab/MMC & 8 & $3 / 0.6$ & $15 / 1.0$ & 9 & $\begin{array}{l}\text { Preoperative leakage_-postoperative localised implant exposure, secondary } \\
\text { conjunctival advancement }\end{array}$ \\
\hline 50 & $\mathrm{~m}$ & Trab/MMC, 5-FU injection & 11 & $5 / 0.2$ & $9 / 0.2$ & 11 & $\begin{array}{l}\text { Preoperative leakage - postoperative corneal dellen, antiglaucomatous topical } \\
\text { medication }\end{array}$ \\
\hline
\end{tabular}

dissected for CGM implantation. A small $(6 \times 2 \mathrm{~mm})$ or large $(12 \times 1 \mathrm{~mm}) \mathrm{CGM}$ implant was chosen, depending on the individual bleb morphology. This was compressed using a flat forceps and placed subconjunctivally just above the scleral flap to exert counter-pressure on the flap. The large CGM version was always folded before implantation. The conjunctiva was closed by a running vicryl 8-0 suture.

All patients with bleb leakage displayed avascular thin blebs. The avascular leaking area of the conjunctiva was resected and the neighbouring conjunctiva was readapted by mobilisation after subconjunctival placement of CGM.

Postoperative topical treatment consisted of steroid/antibiotics ointment application $(3 \times$ daily $)$.

\section{Statistics}

Descriptive statistics were performed using Prism V.5 (GraphPad, USA). Differences between preoperative and postoperative IOP and visual acuity (as logMAR) were tested for significance using the paired test $(\mathrm{p}<0.05)$.

\section{RESULTS}

\section{IOP and visual acuity}

Mean preoperative IOP was $4.4 \pm 1.4 \mathrm{~mm} \mathrm{Hg}$ and significantly $(\mathrm{p}<0.0001)$ increased to $9.9 \pm 2.8 \mathrm{~mm} \mathrm{Hg}$ at the end of the follow-up. Median follow-up was 6 months with a range of 2-18 months.

In 9 of the 12 patients visual acuity improved following the procedure while in three patients visual acuity remained unchanged (table 1). The improvement in visual acuity as measured by $\log$ MAR (preop $0.63 \pm 0.33$ vs postop $0.31 \pm 0.26$ ) was significant $(p=0.0012)$.

\section{Complications}

In an elder female patient, massive choroidal detachment persisted despite a temporary increase in IOP of up to $11 \mathrm{~mm} \mathrm{Hg}$. In this case, choroidal detachment finally disappeared after sealing the scleral fistula with a pericard patch.
In one male patient with ocular hypotony and bleb leakage CGM exposure was seen 2 weeks after surgery. As reepithelialisation occurred slowly (figure 1A), we decided on secondary conjunctival closure 3 months after CGM implantation by a small rotational conjunctival flap (figure 1B). To clean the exposed surface of debris, we intraoperatively removed a thin superficial layer of the CGM implant displaying only superficial immunostaining for $\alpha$ smooth muscle actin (clone 1A4, DAKO, Denmark) and collagen III (clone HWD1.1, DCS, Germany) and both superficial and deep immunostaining (figure 2) for fibronectin (clone 568, Leica, UK).

The other patient with ocular hypotony and leakage developed corneal dellen near to the subconjunctivally placed CGM (figure 3) and was treated with artificial tears.

Choroidal folds or choroidal detachment completely resolved in 9 of the 12 patients.

Two patients required pressure-reducing topical medication at the end of follow-up (table 1).

\section{DISCUSSION}

CGM engineering offers new surgical perspectives in different fields of medicine where strong wound healing may cause functional and morphologic problems. ${ }^{15}$ In penetrating glaucoma surgery, the failure rate is mainly caused by subconjunctival fibrosis. ${ }^{16}$ Thus, the introduction of intraoperative and postoperative application of antimetabolites has dramatically increased the long-term efficacy of trabeculectomy. ${ }^{17} 18$ The down side of the application of antimetabolites can be sightthreatening bleb complications including bleb leakage and massive ocular hypotony.

Porous biodegradable CGM have been used as a subconjunctival spacer during primary trabeculectomy in order to modify filtering bleb scarring and to control early transscleral filtration around the flap. The three-dimensional scaffold of the implant should protect against tractive scarring by limiting the density of contractile cells and by inducing a regular ingrowth of fibroblasts within the scaffold. ${ }^{9-11}$ First prospective randomised clinical studies are somewhat contradictory, ${ }^{19} 20$ and from our own 
(a)

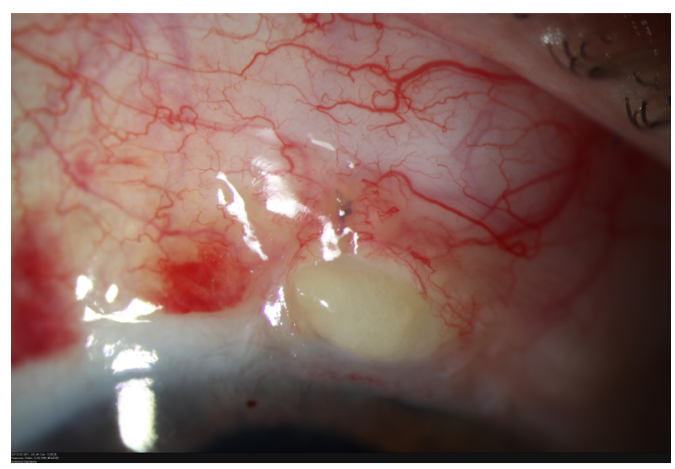

(b)

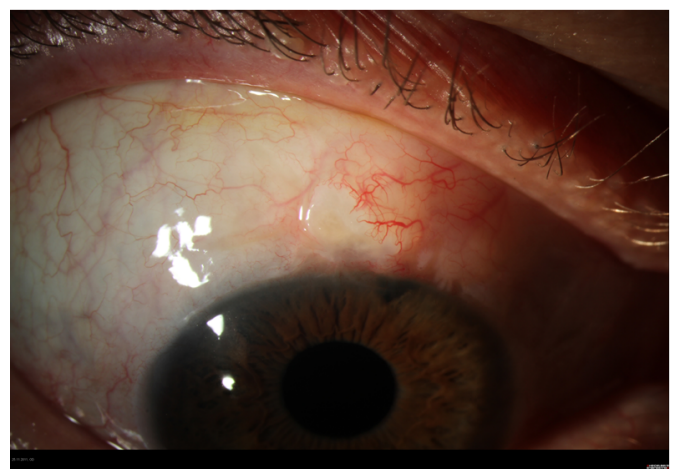

Figure 1 (A) Exposure of the collagen glycosaminoglycan matrices (CGM) implant in a patient following partial bleb excision because of bleb leakage and ocular hypotony. Incomplete epithelial closure occurred very slowly over a period of 3 months. (B) Same patient as presented in (A): Secondary conjunctival advancement by a rotational flap had been performed together with an excision of a superficial layer of the CGM to cover the CGM implant completely. 2 months later the intraocular pressure is around $10 \mathrm{~mm} \mathrm{Hg}$ and the bleb shows no leakage or dehiscence. Access the article online to view this figure in colour.

clinical study it appears that the biodegradable CGM cannot completely match the efficacy of mitomycin $\mathrm{C}$ in achieving low target pressure levels. ${ }^{21}$

Clinical experience shows that the CGM implant exerts counter pressure on the scleral flap, thus limiting transscleral filtration in the early postoperative period. Accordingly, most

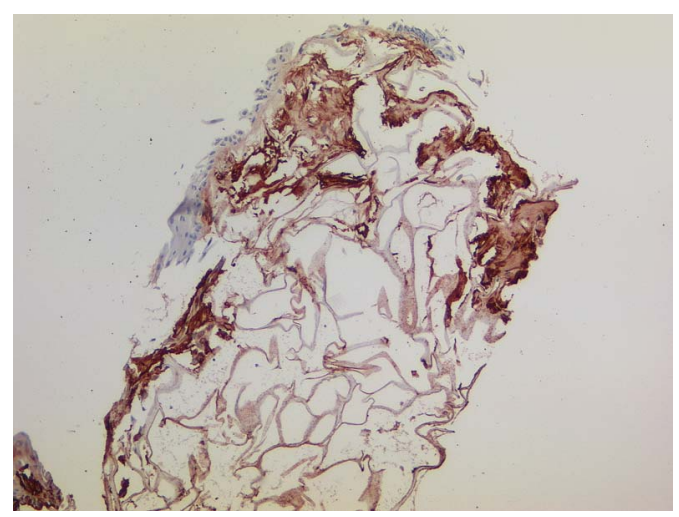

Figure 2 Fibronectin immunostaining of the superficial excision from the exposed porous collagen glycosaminoglycan matrices (CGM) implant presented in figure 1. Immunreactivity is not only detectable in the margins, but also in the deeper layers. On the left side, epithelial cells are covering the CGM implant. Access the article online to view this figure in colour.

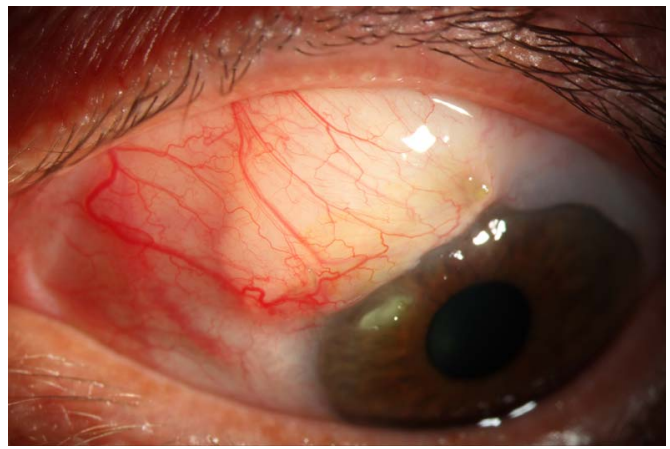

Figure 3 Corneal dellen in the $10 o^{\prime}$ clock position owing to the relatively prominent filtering bleb 4 weeks after secondary collagen glycosaminoglycan matrices (CGM) implantation. The $12 \mathrm{~mm}$-sized CGM implant can be suspected under the conjunctiva. Access the article online to view this figure in colour.

glaucoma surgeons using the ologen implant during trabeculectomy recommend making only one scleral flap suture.

As CGM implants are also thought to promote angiogenesis and to counteract the ischaemic complications of the antimetabolite mitomycin C following glaucoma surgery, ${ }^{8}$ secondary implantation of collagen matrix implant in post-trabeculectomy eyes with ocular hypotony should present a useful combined mechanical and biological approach to limiting overfiltration.

Histopathology of partially excised portions of the CGM 3 months after implantation revealed myofibroblasts surrounding and slowly invading the scaffold. We hypothesise that a secondary tissue reaction around the matrix scaffold contributes to the long-term increase in IOP. A recent clinical study presenting data on the surgical outcome of mitomycin C-soaked collagen matrix implant in trabeculectomy ${ }^{22}$ showed encapsulated blebs at a more rapid pace in larger amounts compared with conventional trabeculectomy partially supporting the observation of an intra-bleb tissue reaction around the matrix.

An animal study about the treatment of a mitomycin C-altered conjunctiva by CGM use has demonstrated by histology that an inflammatory response around and inside the matrix had taken place at day 7 postoperatively and that the use of the CGM had improved vascularity in a previously avascular area at week $4 .{ }^{8}$ However, the authors found the time needed for epithelial closure of an experimental wound or attainment of final conjunctival thickness to be independent of whether or not CGM was used. Our clinical and histologic observations in a patient with a CGM implant shows that epithelialisation of a conjunctival defect filled with a CGM occurs very slowly compared to the situation in animal trials. ${ }^{11}$ Thus, it would seem advisable to surgically cover the CGM completely by conjunctival mobilisation and not to wait for spontaneous reepithelialisation. Conjunctival advancement ${ }^{23-25}$ still remains essential to substitute for the leaking conjunctiva of the filtering bleb, but this can easily be combined with CGM implantation to reduce contractile shrinkage within the bleb.

An obvious short-term advantage of our approach is the absence of massive IOP peaks following the implantation of CGM, enabling this intervention to be performed as an outpatient procedure. In contrast, the risk arises of inducing a sudden pressure spike when viscoelastics are injected into the anterior chamber or, to a lesser extent, when sutures are placed transconjunctivally. In the midterm or long term, the risk of bleb fibrosis needs to be critically assessed, because resorption of the CGM matrix may be associated with a regression of the bleb volume, and thereby, affect the functional outcome. However, reliable data on long-term efficacy are still lacking. 
Contributors All authors contributed equally to the manuscript. TD as the main author wrote the manuscript.

Competing interests The authors' institution received study support for another registered trial (clinical trial.gov number NCT 01174420) concerning the CGM implant by P.J. Dahlhausen\&Co, Gmbh, Cologne, Germany.

Ethics approval Retrospective case series.

Provenance and peer review Not commissioned; externally peer reviewed.

\section{REFERENCES}

1 Smith MF, Magauran RG III, Betchkal J, et al. Treatment of postfiltration bleb leaks with autologous blood. Ophthalmology 1995;102:868-71.

2 Burnstein A, WuDunn D, Ishii Y, et al. Autologous blood injection for late-onset filtering bleb leak. Am J Ophthalmol 2001;132:36-40.

3 Haynes WL, Alward WL. Rapid visual recovery and long-term intraocular pressure control after donor scleral patch grafting for trabeculectomy-induced hypotony maculopathy. J Glaucoma 1995;4:200-1.

4 Harizman N, Ben-Cnaan R, Goldenfeld M, et al. Donor scleral patch for treating hypotony due to leaking and/or overfiltering blebs. J Glaucoma 2005;14:492-6.

5 Higashide T, Tagawa S, Sugiyama K. Intraoperative Healon5 injection into blebs for small conjunctival breaks created during trabeculectomy. I Cataract Refract Surg 2005:31:1279-82.

6 Shirato S, Maruyama K, Haneda M. Resuturing the scleral flap through conjunctiva for treatment of excess filtration. Am J Ophthalmol 2004;137:173-4.

7 Panday M, Shantha B, George R, et al. Outcomes of bleb excision with free autologous conjunctival patch grafting for bleb leak and hypotony after glaucoma filtering surgery. J Glaucoma 2011;20:392-7.

8 Peng YJ, Pan CY, Hsieh YT, et al. The application of tissue engineering in reversing mitomycin C-induced ischemic conjunctiva. J Biomed Mater Res Part A 2012;100A:1126-35

9 Chen HS, Ritch R, Krupin T, et al. Control of filtering bleb structure through tissue bioengineering: an animal model. Invest Ophthalmol Vis Sci 2006;47:5310-14.

10 Hsu WC, Spilker MH, Yannas IV, et al. Inhibition of conjunctival scarring and contraction by a porous collagen-glycosaminoglycan implant. Invest Ophthalmol Vis Sci 2000;41:2404-11.

11 Hsu WC, Ritch R, Krupin T, et al. Tissue bioengineering for surgical bleb defects: an animal study. Graefes Arch Clin Exp Ophthalmol 2008;246:709-17.
12 Aptel F, Dumas S, Denis P. Ultrasound biomicroscopy and optical coherence tomography imaging of filtering blebs after deep sclerectmoy with new collagen implant. Eur J Ophthalmol 2009;19:223-30.

13 Rosentreter A, Schild AM, Dinslage S, et al. Biodegradable implant for tissue repair after glaucoma drainage device surgery. J Glaucoma 2012;21:76-8.

14 Dada T, Kusumesh R, Bali SJ, et al. Trabeculectomy with combined use of subconjunctival collagen implant and low-dose mitomycin C. J Glaucoma 2012 (epub ahead).

15 Yannas IV, Tzeranis DS, Harley BA, et al. Biologically active collagen-based scaffolds: advances in processing and characterization. Phil Trans $R$ Soc $A$ 2010;368:2123-39.

16 Hitchings RA, Grierson I. Clinico pathological correlation in eyes with failed fistulising surgery. Trans Ophthalmol Soc UK 1983;103:84-8.

17 Khaw PT, Doyle JW, Sherwood MB, et al. Prolonged localized tissue effects from 5-minute exposures to 5 -fluorouracil and mitomycin C. Arch Ophthalmol 1993;111:263-7.

18 Cordeiro MF, Reichel MB, Gay JA, et al. Transforming growth factor-beta1, -beta2, and -beta3 in vivo: effects on normal and mitomycin C-modulated conjunctival scarring. Invest Ophthalmol Vis Sci 1999;40:1975-82.

19 Papaconstantinou D, Georgalas I, Karmiris E. Trabeculectomy with OloGen vs trabeculectomy for the treatment of glaucoma: a pilot study. Acta Ophthalmol 2010;88:80-5.

20 Cillino S, DiPace F, Cillino G, et al. Biodegradable collagen matrix implant vs mitomycin- $\mathrm{C}$ as an adjuvant in trabeculectomy: a 24-month, randomized clinical trial. Eye 2011;25:1598-606.

21 Rosentreter A, Schild AM, Jordan JF, et al. A prospective randomised trial of trabeculectomy using mitomycin C vs an ologen implant in open angle glaucoma. Eye 2010;24:1449-57.

22 Min JK, Kee CW, Sohn SW, et al. Surgical outcome of Mitomycin C-soaked Collagen Matrix Implant in Trabeculectomy. J Glaucoma 2013 (Epub ahead).

23 Budenz DL, Chen PP, Weaver YK. Conjunctival advancement for late-onset filtering bleb leaks: indications and outcomes. Arch Ophthalmol 1999;117:1014-19.

24 Tannenbaum DP, Hoffman D, Greaney MJ, et al. Outcomes of bleb excision and conjunctival advancement for leaking or hypotonous eyes after glaucoma filtering surgery. Br J Ophthalmol 2004;88:99-103.

25 Bochmann F, Azuara-Blanco A. Interventions for late trabeculectomy bleb leak. Cochrane Database Syst Rev 2012;9:CD006769. 